\title{
Repeating head-on collisions in an optical trap and the evaluation of spin-dependent interactions among neutral particles
}

\author{
Z B Li, Z F Chen, Y Z He, C G Baot \\ State Key Laboratory of Optoelectronic Materials and Technologies, and Department \\ of Physics, Sun Yat-Sen University, Guangzhou, 510275, P.R. China \\ E-mail: stsbcg@mail.sysu.edu.cn
}

\begin{abstract}
A dynamic process of repeating collisions of a pair of trapped neutral particles with weak spin-dependent interaction is designed and studied. Related theoretical derivation and numerical calculation have been performed to study the inherent coordinate-spin and momentum-spin correlation. Due to the repeating collisions the effect of the weak interaction can be accumulated and enlarged, and therefore can be eventually detected. Numerical results suggest that the $\mathrm{Cr}-\mathrm{Cr}$ interaction, which has not yet been completely clear, could be thereby determined. The design can be in general used to determine various interactions among neutral atoms and molecules, in particular for the determination of very weak forces.
\end{abstract}

PACS numbers: $34.90 .+\mathrm{q}, 34.50 . \mathrm{Cx}, 03.75 . \mathrm{Mn}$

$\ddagger$ The corresponding author 


\section{Introduction}

It is well known that the study of scattering is an important way to understand the interactions among particles. Experimentally, the incident particles with certain momentum would hit a target to initiate the scattering. The accuracy of experimental data depends on a precise control of bombarding energy. For charged incident particles, the initial momenta are usually imposed by electromagnetic forces which can be precisely tuned. For neutral incident particles, the initial momenta are in general difficult to control precisely. Furthermore, the interactions among neutral atoms or molecules are in general weak (say, the van der Waals force). Therefore, the precise determination of these interactions is difficult. Consequently, these interactions are usually determined in an indirect way (say, comparing related experimental data of spectroscopy and/or thermodynamics of a many-body system with theoretical results based on a model with a given set of parameters). In this way the associated theoretical calculations are usually complicated, and uncertainty might exist.

On the other hand, the trapping of neutral atoms has been realized via optical traps since 1998 [1, 2]. This techniques is in progress and fewer atoms can be trapped recently [3]. It might open a new way for studying the interactions. In this paper, an idea is proposed and related theoretical calculation is performed to show how the scattering with precisely controllable initial status can be realized in a trap. It turns out that, as we shall see, the collisions would occur repeatedly. Thereby the effect of each individual collision can be accumulated and therefore enlarged. This might lead to a better understanding of very weak interactions among neutral particles.

Since the pioneer experiment by Greismaier, et. al. [4], the Bose-Einstein condensations of atoms with a larger spin (say, ${ }^{52} \mathrm{Cr}$ ) become a hot topic. These condensates are a new kind of matter aggregation having the magnetic dipole-dipole interaction $V_{d d}$ more than twenty times stronger than that of the alkalis family.

$$
V_{d d}=\frac{C_{d}}{r^{3}}\left[\mathbf{F}_{1} \cdot \mathbf{F}_{2}-3 \frac{\left(\mathbf{F}_{1} \cdot \mathbf{r}\right)\left(\mathbf{F}_{2} \cdot \mathbf{r}\right)}{r^{2}}\right],
$$

where the strength $C_{d}=\mu_{0} \mu_{B}^{2} g_{F}^{2} /(4 \pi)$ with $\mu_{0}$ being the magnetic permeability of vacuum, $\mu_{B}$ the Bohr magneton, and $g_{F}$ the Landé $g$ factor, $\mathbf{F}_{i}$ the operator of the spin of the $i$-th atom, and $\mathbf{r}=\mathbf{r}_{2}-\mathbf{r}_{1}$. Consequently, the spatial and spin degrees of freedom are coupled so that the conversion of spin angular momentum into orbital angular momentum can be realized. Thereby new physical phenomena (say, rotonlike behavior) might appear [5, 6]. In addition to the long range $V_{d d}$, the short range interaction is also spin-dependent and, for low-energy systems, can be in general written as $V_{\delta}=\delta\left(\mathbf{r}_{1}-\mathbf{r}_{2}\right) \sum_{S} g_{S} \mathfrak{P}^{S}$, where $S$ is the total spin, $g_{S}$ is the strength related to the $s$-wave scattering length of the $S$-spin channel, and $\mathfrak{P}^{S}$ the projector of the $S$ channel. $g_{S}$ is nonzero only if $S$ is even. $g_{2}, g_{4}$, and $g_{6}$ are known while $g_{0}$ has not yet [7, 8, 9, 10]. However, many features of the condensate depend strongly on $g_{0}$ (say, the phase-diagrams [10, 11, 12, 13] and the spin-evolutions [14]). Therefore, the determination of $g_{0}$ is important for a thorough and clear description of this condensate. 
As an application of our idea of repeating collisions in a trap, the ${ }^{52} \mathrm{Cr}$ atoms have been chosen as an example to see to what extent the interaction can be thereby clarified.

In the beginning, two narrow and deep potentials $\frac{1}{2} m \omega_{a}^{2}|\mathbf{r} \pm \mathbf{a}|^{2}$ are preset at $\pm \mathbf{a}$ (say, two magnetic traps), where $\mathbf{a}$ is lying along the positive $Z$-axis. Each potential contains a $\mathrm{Cr}$ atom in the ground state of the parabolic confinement. Both atoms are polarized but in reverse directions. The upper (lower) atom has spin-component $\mu=3(-3)$. Thus the magnetization $M_{S}$ of the system is zero, and the two atoms are localized. Instantly, the two preset potentials are replaced by a broader potential $\frac{1}{2} m \omega^{2} r^{2}$ (say, an optical trap) centering at the origin. Then, the previously localized atoms begin to evolve. Since $M_{S}=0$, the $S=0$ component must be included and will be affected by $g_{0}$. Therefore, by observing the evolution, the knowledge on $g_{0}$ might be extracted.

\section{Hamiltonian and initial state}

Introduce $\mathbf{R}=\left(\mathbf{r}_{1}+\mathbf{r}_{2}\right) / 2$ and $\mathbf{r}=\mathbf{r}_{2}-\mathbf{r}_{1}$ for the c.m. and relative motions. Introduce $\hbar \omega$ and $\sqrt{\hbar / m \omega}$ as the units of energy and length, respectively. The symmetrized and normalized initial state

$$
\begin{aligned}
\Psi_{I}= & \frac{1+P_{12}}{\sqrt{2}}\left[\left(\frac{2 \alpha}{\pi}\right)^{3 / 4} e^{-\alpha R^{2}}\right] \\
& \cdot\left[\left(\frac{\alpha}{2 \pi}\right)^{3 / 4} e^{-\alpha\left(r^{2} / 4+a^{2}+r a \cos \theta\right)}\right] \chi_{3}(1) \chi_{-3}(2),
\end{aligned}
$$

where $P_{12}$ denotes an interchange of 1 and $2, \alpha=\omega_{a} / \omega, \theta$ is the angle between $\mathbf{r}$ and the $Z$-axis, $a=|\mathbf{a}|$, and $\chi_{\mu}$ is a spin-state of an atom with component $\mu$. Then the evolution is governed by the Hamiltonian

$$
H_{\text {evol }}=H_{R}+H_{r}+V_{12} \text {, }
$$

where $H_{R} \equiv-\frac{1}{4} \nabla_{R}^{2}+R^{2}$ and $H_{r} \equiv-\nabla_{r}^{2}+\frac{1}{4} r^{2}$, and $V_{12}=V_{\delta}+V_{d d}$. The eigenstates of $H_{R}$ and $H_{r}$, denoted as $\bar{\phi}_{N L}(R) Y_{L M}(\hat{R})$ and $\phi_{n l}(r) Y_{l m_{l}}(\hat{r})$, are just the harmonic oscillator states. The eigenstates of $H_{r}+V_{12}$ can be expanded in terms of basis functions as

$$
\psi_{i}^{J}=\sum_{\gamma} C_{i \gamma}^{J} \phi_{n l}(r)(l S)_{J},
$$

where $\gamma$ represents the set $n, l$, and $S,(l S)_{J}$ denotes the coupling of $l$ and $S$ into the total angular momentum $J, i$ is just an index of the $J$-series. Due to $V_{d d}, l$ and $S$ are not conserved, but $J$ is. Due to the boson statistics, $l+S$ must be even.

It turns out that $V_{12}$ is rather weak in our case. Consequently, each eigenstate of $H_{r}+V_{12}$ is close to an eigenstate of $H_{r}$. This fact leads to a great reduction of necessary basis functions in the expansion. When a set of basis functions has been chosen, the associated matrix elements of $H_{r}+V_{12}$ can be derived as shown in the appendix. Carrying out the diagonalization, the coefficients $C_{i \gamma}^{J}$ and the corresponding eigenenergy $E_{i}^{J}$ can be obtained. In terms of $\bar{\phi}_{N L}$ and $\psi_{i}^{J}$, the initial state can be rewritten as

$$
\Psi_{I}=\sum_{N} B_{N} \bar{\phi}_{N, 0}(R) Y_{00}(\hat{R}) \sum_{J, i} b_{i}^{J} \psi_{i}^{J} .
$$


Making use of Eq. (4), and equating (5) and (2), it is straight forward to obtain the coefficients $B_{N}$ and $b_{i}^{J}$.

\section{Time-dependent density and the repeating collisions}

The time-dependent solution for the evolution is

$$
\begin{aligned}
\Psi(t)= & e^{-i H_{\text {evol }} \tau} \Psi_{I} \\
= & \frac{1}{\sqrt{4 \pi}} \sum_{N} B_{N} e^{-i\left(2 N+\frac{3}{2}\right) \tau} \bar{\phi}_{N, 0}(R) \\
& \cdot \sum_{J, i} b_{i}^{J} e^{-i E_{i}^{J} \tau} \psi_{i}^{J},
\end{aligned}
$$

where $\tau=\omega t$.

From Eq. (6), all the information on the evolution can be extracted. The main feature of the evolution is the occurrence of repeated collisions, as we shall see, and the effect of interaction on each collision can be accumulated and therefore enlarged. Thereby the strength $g_{0}$ can be evaluated.

We firstly extract the time-dependent density from $\Psi(t)$ as

$$
\begin{aligned}
\rho(r, \theta, t) \equiv & 2 \pi r^{2} \int d \mathbf{R} \Psi^{*}(t) \Psi(t) \\
= & 2 \pi r^{2} \sum_{J i \gamma, J^{\prime} i^{\prime} \gamma^{\prime}} \delta_{S^{\prime}, S} \cos \left[\left(E_{i^{\prime}}^{J^{\prime}}-E_{i}^{J}\right) \tau\right] \\
& \cdot b_{i^{\prime}}^{J^{\prime}} C_{i^{\prime} \gamma^{\prime}}^{J^{\prime}} b_{i}^{J} C_{i \gamma}^{J} \sum_{M_{S}} C_{l^{\prime},-M_{S} ; S M_{S}}^{J^{\prime}, 0} C_{l,-M_{S} ; S M_{S}}^{J, 0} \\
& \cdot \phi_{n^{\prime} l^{\prime}}(r) \phi_{n l}(r)\left|Y_{l^{\prime},-M_{S}}\right|\left|Y_{l,-M_{S}}\right|
\end{aligned}
$$

where the Clebsch-Gordan coefficients have been introduced. It satisfies

$$
1=\int d r \sin \theta d \theta \rho(r, \theta, t) .
$$

When the parameters are given as $\omega=2000 \times 2 \pi, \alpha=1.5, a=2$, and $g_{0}=-g_{6} / 2$, the variation of $\rho$ in the earliest stage of evolution is shown in Fig. 1. Since $\rho$ does not depend on the azimuthal angle and $\rho(r, \pi-\theta, t)=\rho(r, \theta, t)$, it is sufficient to be plotted only on a quarter of $z-x$ plane, where $z$ and $x$ are the components of $\mathbf{r} / 2$. Since the c.m. is always distributed close to the origin, we have roughly $\mathbf{r}_{2} \simeq \mathbf{r} / 2$ and $\mathbf{r}_{1} \simeq-\mathbf{r} / 2$. Thus the distribution of an atom can be understood from Fig. 1. When the evolution begins, the two atoms located at opposite ends of the broad potential collide straightly with each other (see 11a and 10). When $t \approx \pi / 2 \omega(\mathbb{1} \mathrm{r})$, the two atoms keep close to each other with a distance $\sim 1.6$, and they are both distributed around the center. Afterward, the atoms begin to separate (11 is very similar to $1 \mathrm{~b}$ ). When $t=\pi / \omega$, the profile (not yet shown) is very similar to 1 a. Thus the first round of head-on collision has been completed, and the second round will begin successively. If we remove $V_{12}$ from $H_{\text {evol }}$, the factor $\left(E_{i^{\prime}}^{J^{\prime}}-E_{i}^{J}\right)$ in Eq. (7) would become an integral multiple of $\hbar \omega$, thereby the above process would be exactly periodic with the period $2 \pi / \omega$. In fact, the collision as 


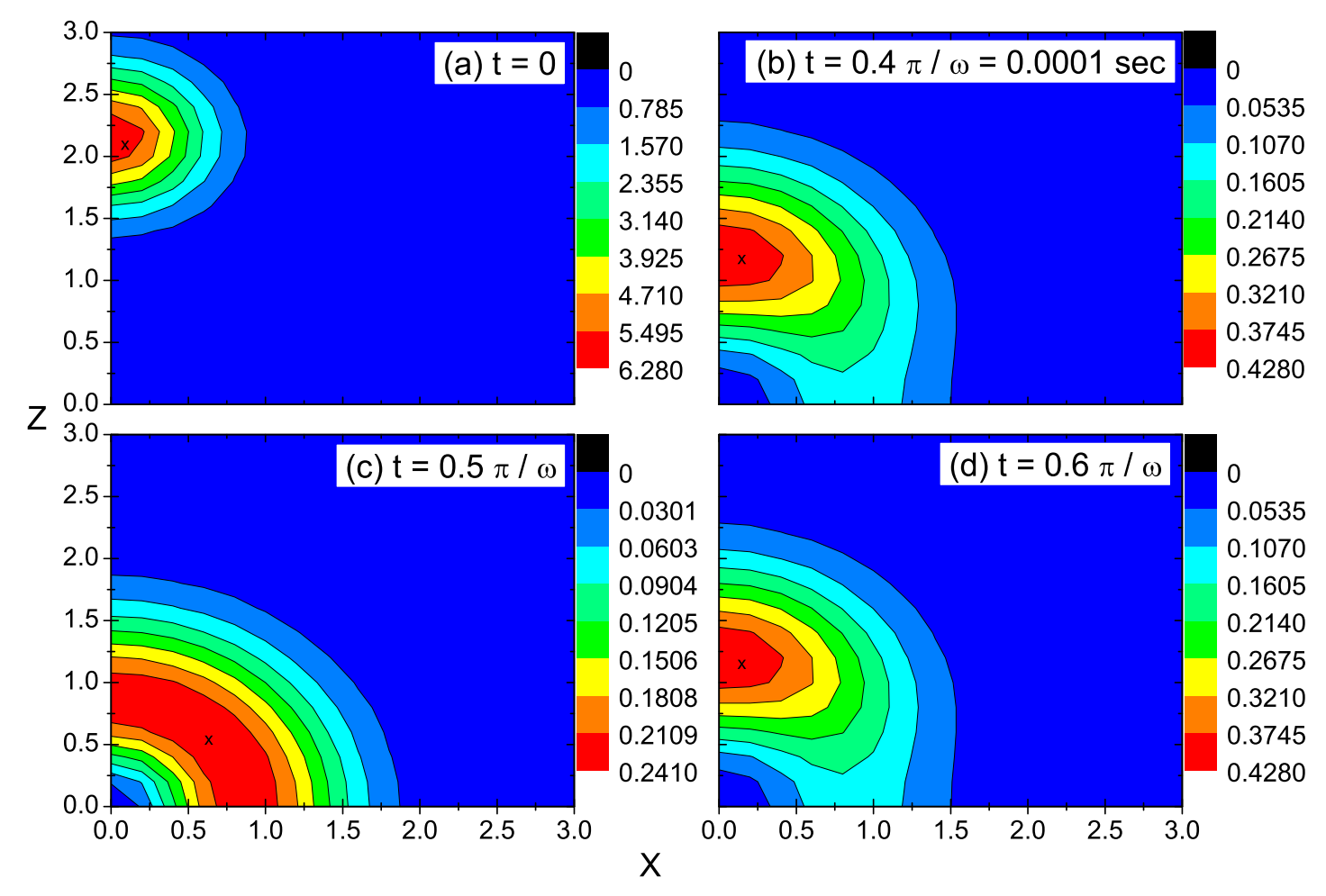

Figure 1. (Color online) $\rho(r, \theta, t)$ plotted in the $z-x$ plane, where $z$ and $x$ are the components of $\mathbf{r} / 2$. $t$ is given at 4 values in the interval ( 0 to $0.6 \pi / \omega=0.00015 \mathrm{sec}$ ). In the panel, the area with the largest $\rho(r, \theta, t)$ is marked by a $\times$. The parameters are given as $\omega=2000 \times 2 \pi, \alpha=1.5,|\mathbf{a}|=2 \sqrt{\hbar / m \omega}=311 \mathrm{~nm}$, and $g_{0}=-g_{6} / 2$.

shown in Fig. 1 is essentially determined by $H_{r}$. In the early stage, $V_{12}$ causes only a very small perturbation. However, as we shall see, the effect of each collision can be accumulated when the time goes on.

The number of collisions that the two atoms have experienced within $t$ is $\sim t \omega / \pi$. When $t=0.07 \mathrm{sec}$ and $\omega=2000 \times 2 \pi$, this number is $\approx 280$. When $t$ is close to $0.07 \mathrm{sec}$, $\rho$ is shown in Fig. 2. At the first glance, $2 a$ is similar to 1 a. However, the peak in $2 a$ is considerably lower than that of 1 a implying that the atoms are not well localized as in the beginning. In fact, $\rho$ has spread widely in $2 \mathrm{a}$ and contains a smooth peak at $x=1.6$ and $z=1.4$ (this smooth peak is too low to be seen in the figure). The two atoms are closer to each other in $2 \mathrm{~b}$ than in $1 \mathrm{~b}$. The density varies more vigorously along $\theta$ in $2 \mathrm{c}$ than in 1 c. 2 $\mathrm{b}$ and $2 \mathrm{~d}$ do not have the approximate similarity as shown previously in $1 \mathrm{~b}$ and $1 \mathrm{~d}$. From $2 \mathrm{a}$ and $2 \mathrm{f}$, we know that a round of collision has been completed in the interval from 0.07 to $0.07+\pi / \omega$. The evolution in this round is explicitly different from that in the first round due to the accumulated effect of $V_{12}$.

The accuracy of the above numerical results depends on the number of basis functions, which is determined by the scopes of $n$ (from 0 to $n_{\max }$ ), $l$ (from 0 to $l_{\max }$ ), and $N$ (from 0 to $N_{\max }$ ). When $n_{\max }=N_{\max }=12$ and $l_{\max }=14$, the associated results are found to be nearly the same as those by using $n_{\max }=N_{\max }=10$ and $l_{\max }=12$. Thus we believe that the former choice is sufficient. 


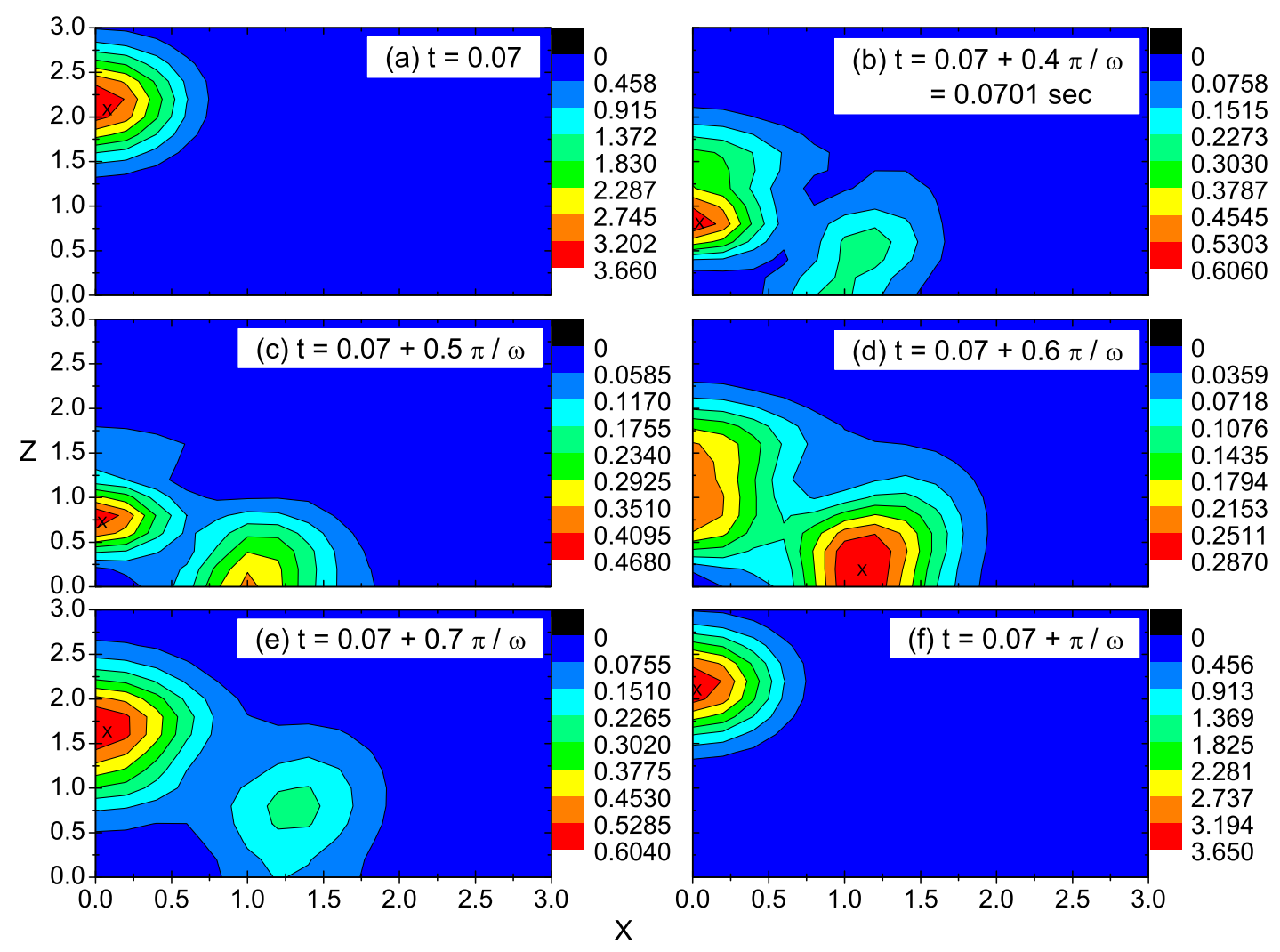

Figure 2. (Color online) The same as Fig. 1 but $t$ is given in the interval (0.07 to $0.07+\pi / \omega)$.

\section{Time-dependent probability of the spin-component and the evaluation}

Due to the spin-dependent interaction, spin-flips will occur during the evolution. From Eq. (6) the time-dependent probability of the spin-component of an atom in $\mu$ is

$$
\begin{aligned}
P_{\mu}(t)= & \sum_{J i \gamma, J^{\prime} i^{\prime} \gamma^{\prime}} \delta_{n^{\prime} n} \delta_{l^{\prime} l} \cos \left[\left(E_{i^{\prime}}^{J^{\prime}}-E_{i}^{J}\right) \tau\right] \\
& \cdot b_{i^{\prime}}^{J^{\prime}} C_{i^{\prime} \gamma^{\prime}}^{J^{\prime}} b_{i}^{J} C_{i \gamma}^{J} \sum_{\lambda}(2 \lambda+1) \sqrt{\left(2 S^{\prime}+1\right)(2 S+1)} \\
& \cdot W\left(l 3 J^{\prime} 3 ; \lambda S^{\prime}\right) W(l 3 J 3 ; \lambda S) \\
& \cdot C_{\lambda,-\mu ; 3 \mu}^{J^{\prime}, 0} C_{\lambda,-\mu ; 3 \mu}^{J, 0},
\end{aligned}
$$

where both the Clebsch-Gordan and Wigner coefficients [15] have been introduced. To show the convergency of numerical calculation, the dependence of $P_{\mu}(t)$ on the number of basis functions is given in Tab. 1.

It is clear from the table that, when $n_{\max }=N_{\max }=12$ and $l_{\max }=14$ as we have adopted, qualitatively accurate results can be obtained.

Due to the symmetry inherent in $\Psi_{I}$ and in $H_{\text {evol }}, P_{-\mu}(t)=P_{\mu}(t)$. The variation of $P_{3}(t)$ against $t$ is shown in Fig. 3, where $g_{0}$ is given at three values. It is recalled that the first round of collision ends at $t=\pi / \omega=0.00025 \mathrm{sec}$. In this very short time, the change 
Table 1. $P_{3}(t)$ at three values of $t$ (in sec). The parameters are given as $\omega=1000 \times 2 \pi$, $\alpha=3,|\mathbf{a}|=2$, and $g_{0}=-g_{6} / 2$. The number of basis functions depends on $n_{\max }$ and $l_{\max }\left(N_{\max }=n_{\max }\right.$ is assumed $)$ listed in the first row.

\begin{tabular}{lrrr}
\hline$n_{\max }$ and $l_{\max }$ & 10 and 12 & 12 and 14 & 14 and 16 \\
\hline$P_{3}(0.02)$ & 0.4908 & 0.4914 & 0.4914 \\
$P_{3}(0.06)$ & 0.4728 & 0.4733 & 0.4734 \\
$P_{3}(0.10)$ & 0.4751 & 0.4758 & 0.4760 \\
\hline
\end{tabular}

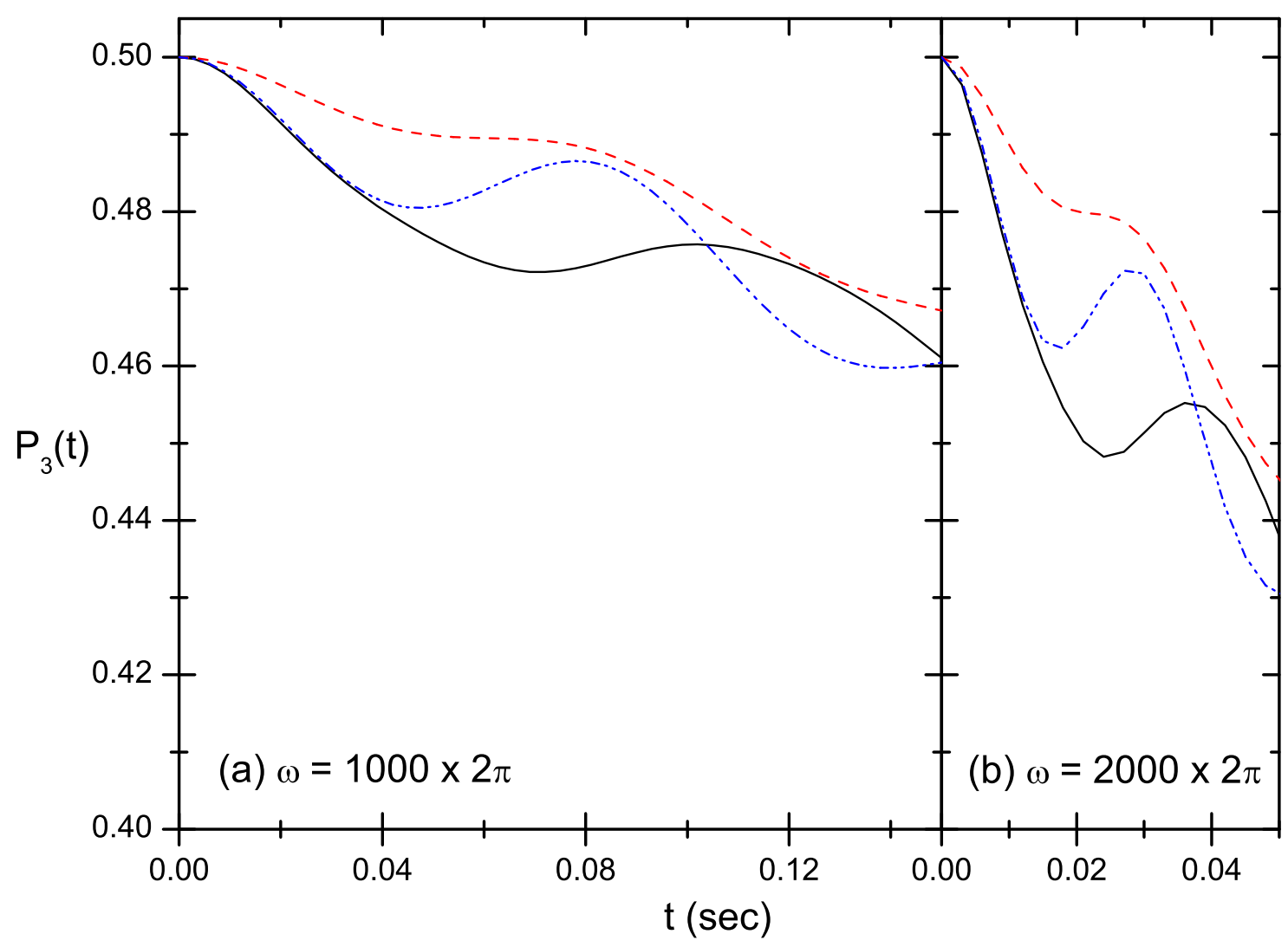

Figure 3. (Color online) The evolution of $P_{3}(t)$. The solid, dash, and dash-dot-dot curves have $g_{0}=-g_{6} / 2,0$, and $g_{6} / 2$, respectively. In [3a, $\omega=1000 \times 2 \pi, \alpha=3$, $|\mathbf{a}|=2$, while in $3 \mathrm{~b}, \omega=2000 \times 2 \pi, \alpha=1.5,|\mathbf{a}|=2$.

of $P_{3}(t)$ is negligible. However, after hundreds of repeating collisions, the dependence of $P_{3}(t)$ on $g_{0}$ can be clearly detected as shown in the figure. Therefore, $P_{\mu}(t)$ can be used to evaluate $g_{0}$. In particular, the dependence will become more explicit if $\omega$ is larger (comparing $3 \mathrm{~b}$ with $3 \mathrm{a}$ ). The evolutions of $P_{2}(t)$ and $P_{0}(t)$ are shown in Fig. 4a and $4 \mathrm{~b}$, respectively, where $g_{0}$ is given at seven values. One can see that there is a small peak in 4 appearing in the early stage of evolution if $g_{0}$ is negative. Its height depends on how negative $g_{0}$ is, and it would disappear if $g_{0}$ is positive. Therefore, the existence or not of this peak can be used to judge the sign of $g_{0}$. Together with the height and location of the second peak in Fig. 3 (or the first peak of $4 \mathrm{~b}$ ), the strength $g_{0}$ can be known. 


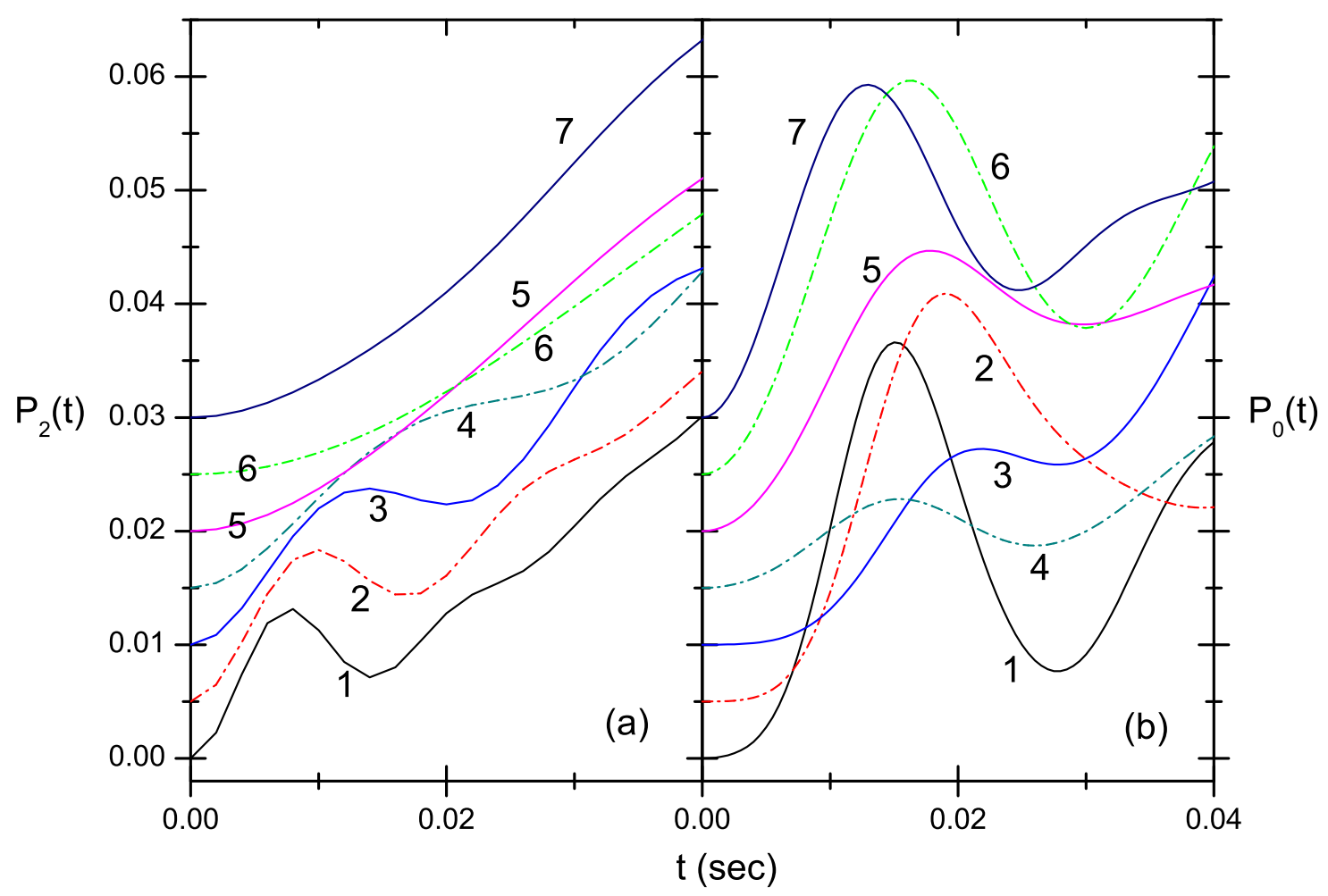

Figure 4. (Color online) The evolution of $P_{2}(t)$ (a) and $P_{0}(t)$ (b). The curves from "1" to " 7 " have $g_{0}$ from $-3 g_{6} / 4$ to $3 g_{6} / 4$, respectively, with an increase $g_{6} / 4$ in each step. Each curve has shifted up by 0.005 more than its adjacent lower neighbor to guide the eyes. The other parameters are the same as in Fig. 1.

Since $V_{d d}$ can alter $l$, the spatial structure can thereby be altered. On the other hand, $V_{d d}$ can also alter $S$; therefore spin-evolutions are also affected. We found that, if $V_{d d}$ is reduced (strengthened), the spin-flips would occur less (more) probable. E.g., when $V_{d d}$ is changed to $\beta V_{d d}$, the values of the first minimum of the solid curve of Fig. 3 $\mathrm{b}$ would be $0.462,0.448$, and 0.298 , respectively, if $\beta=0,1$, and 10 . It implies that a strong dipole force will cause strong spin-flips. This is a notable point. On the other hand, recent experimental progress suggests that condensation of molecules with large permanent dipole moments, such as $\mathrm{OH}$ [16, 17], RbCs [18, KRb [19], and $\mathrm{NH}$ [20, may be achieved. These systems would have very strong dipole interaction, $10^{2}$ or more times stronger than in chromium. Therefore, distinguished phenomena of spins caused by the very strong $V_{d d}$ are expected.

In order to understand better the phenomenon of spin-flips, the momentum-spin correlation is studied in the following. Let the coordinates $\mathbf{r}_{1}$ and $\mathbf{r}_{2}$ be introduced via the Talmi-Moshinsky (T-M) coefficients as

$$
=\sum_{n_{1} l_{1} n_{2} l_{2}} a_{n_{1} l_{1} n_{2} l_{2}}^{N 0 n l l} \hat{\phi}_{n_{1} l_{1}}\left(r_{1}\right) \hat{\phi}_{n_{2} l_{2}}\left(r_{2}\right)\left[Y_{l_{1}}\left(\hat{r}_{1}\right) Y_{l_{2}}\left(\hat{r}_{2}\right)\right]_{l m},
$$

where $l_{1}$ and $l_{2}$ are coupled to $l$ and $m$, and $\hat{\phi}_{n l}\left(r_{i}\right) Y_{l m_{l}}\left(\hat{r}_{i}\right)$ are the eigenstates of 
$H_{r_{i}} \equiv-\frac{1}{2} \nabla_{r_{i}}^{2}+\frac{1}{2} r_{i}^{2}$. The analytical expression of T-M coefficients has been given in the refs. [21, 22, 23]. Inserting Eq. (10) into (6), we have

$$
\begin{aligned}
\Psi(\tau)= & \sum_{n_{1} l_{1}, n_{2} l_{2}, m_{1} m_{2}, \mu \nu} \delta_{m_{1}+m_{2},-\mu-\nu} Y_{n_{1} l_{1}, n_{2} l_{2}, m_{1} m_{2}}^{\mu \nu}(\tau) \\
& \cdot \hat{\phi}_{n_{1} l_{1}}\left(r_{1}\right) \hat{\phi}_{n_{2} l_{2}}\left(r_{2}\right) Y_{l_{1} m_{1}}\left(\hat{r}_{1}\right) Y_{l_{2} m_{2}}\left(\hat{r}_{2}\right) \\
& \cdot \chi_{\mu}(1) \chi_{\nu}(2),
\end{aligned}
$$

where $Y_{n_{1} l_{1}, n_{2} l_{2}, m_{1} m_{2}}^{\mu \nu}(\tau)$ can be known by comparing Eq. (11) with (6) and (10).

Insert Eq. (11) into the normality $\langle\Psi(\tau) \mid \Psi(\tau)\rangle=1$, it is straight forward to obtain the time-dependent probability of finding a particle in $\chi_{\nu}$ state at $\mathbf{r}_{2}$ and $\tau$ as follows

$$
\begin{aligned}
P_{\nu}\left(\mathbf{r}_{2}, \tau\right)= & \sum_{n_{2}^{\prime} l_{2}^{\prime}, n_{2} l_{2}, m_{2}} Z_{n_{2}^{\prime} l_{2}^{\prime}, n_{2} l_{2}, m_{2}}^{\nu}(\tau) \hat{\phi}_{n_{2}^{\prime} l_{2}^{\prime}}\left(r_{2}\right) \hat{\phi}_{n_{2} l_{2}}\left(r_{2}\right) \\
& \cdot Y_{l_{2}^{\prime} m_{2}}^{*}\left(\hat{r}_{2}\right) Y_{l_{2} m_{2}}\left(\hat{r}_{2}\right),
\end{aligned}
$$

where

$$
\begin{aligned}
Z_{n_{2}^{\prime} l_{2}^{\prime}, n_{2} l_{2}, m_{2}}^{\nu}(\tau)= & \sum_{n_{1} l_{1} m_{1} \mu} \delta_{m_{1}+m_{2},-\mu-\nu}\left[Y_{n_{1} l_{1}, n_{2}^{\prime} l_{2}^{\prime}, m_{1} m_{2}}^{\mu \nu}(\tau)\right]^{*} \\
& \cdot Y_{n_{1} l_{1}, n_{2} l_{2}, m_{1} m_{2}}^{\mu \nu}(\tau) .
\end{aligned}
$$

This probability fulfills $\sum_{\nu} \int d \mathbf{r}_{2} P_{\nu}\left(\mathbf{r}_{2}, \tau\right)=1$.

For observing the momentum-spin correlation, we define

$$
\xi_{n l}(p)=\sqrt{\frac{2}{\pi}}(-i)^{l} \int r^{2} d r j_{l}(p r) \hat{\phi}_{n l}(r),
$$

where the spherical Bessel function has been introduced. Then from Eq. (12), we obtain

$$
\begin{aligned}
Q_{\nu}(\mathbf{p}, \tau)= & \sum_{n_{2}^{\prime} l_{2}^{\prime}, n_{2} l_{2}, m_{2}} Z_{n_{2}^{\prime} l_{2}^{\prime}, n_{2} l_{2}, m_{2}}^{\nu}(\tau) \xi_{n_{2}^{\prime} l_{2}^{\prime}}^{*}(p) \xi_{n_{2} l_{2}}(p) \\
& \cdot Y_{l_{2}^{\prime} m_{2}}^{*}(\hat{p}) Y_{l_{2} m_{2}}(\hat{p}),
\end{aligned}
$$

which is the probability of finding a particle in $\nu$ at $\mathbf{p}$ and $\tau$. It fulfills $\sum_{\nu} \int d \mathbf{p} Q_{\nu}(\mathbf{p}, \tau)=1$.

In order to observe $Q_{\nu}$ at a given time $\tau_{c}$, the broad trap has to be suddenly cancelled at $\tau_{c}$. Then, the particles begin to go out and the successive evolution is an expansion and is governed by the Hamiltonian $H_{\text {free }}+V_{12}$, where $H_{\text {free }}=-\frac{1}{2}\left(\nabla_{1}^{2}+\nabla_{2}^{2}\right)$. During the expansion, collisions happen scarcely. We have already seen that the effect of $V_{12}$ within a few collisions is negligible. Therefore, it is safe to neglect $V_{12}$ during the expansion, thus the wave function after $\tau_{c}$ is $\Psi\left(\tau>\tau_{c}\right) \approx e^{-i H_{\text {free }}\left(\tau-\tau_{c}\right)} \Psi\left(\tau_{c}\right) \equiv \Psi_{\text {free }}(\tau)$.

It is straight forward to see that the weight of $\Psi_{\text {free }}(\tau)$ in $\left|\mathbf{p}_{1}, \mathbf{p}_{2}\right\rangle$ is the same as that of $\Psi\left(\tau_{c}\right)$. Therefore, after the cancellation, the probability $Q_{\nu}$ depends only on $\tau_{c}$ but not on $\tau$. Thus, the time of observation $\tau$ is not relevant, but the choice of $\tau_{c}$ is essential.

An example of $Q_{3}\left(\mathbf{p}, \tau_{c}\right)$ in the earliest stage of evolution is given in Fig. 5 a. Curve "1" describes the momentum distribution of the initial h.o. ground state over the $Z$ component of $\mathbf{p}$. When the evolution begins, the distribution shifts to the left as shown 


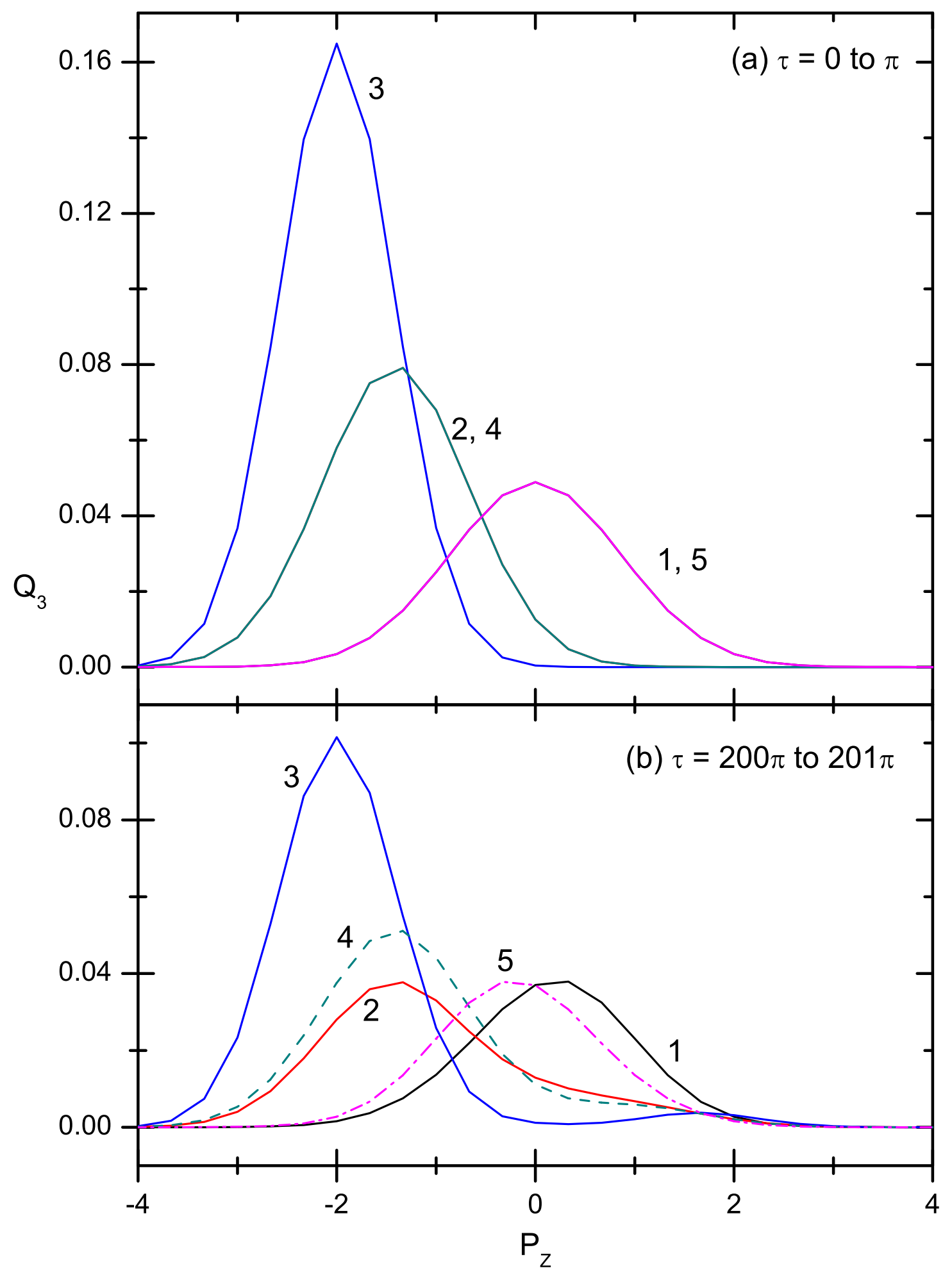

Figure 5. (Color online) The distribution of $Q_{3}\left(\mathbf{p}, \tau_{c}\right)$ over the $Z$-component of $\mathbf{p} . \tau_{c}$ is the time to cancel the trap, and is given at five values as $\tau_{c}=(i-1) \pi / 4+\tau_{X}$, " $i$ " is marked by the associated curve, and $\tau_{X}=0$ (a) and $200 \pi$ (b). The unit of momentum in this paper is $\sqrt{m \hbar \omega}$. The parameters are the same as in Fig. 1. 
by "2". It implies that the component with $\nu=3$ is moving from the initial end toward the center. When $\tau=\pi / 2$, a sharp peak appears at $p_{z}=-2$ as shown by "3". Meanwhile, as we know from Fig. 1k, the particle is close to the center, and it has a maximal momentum pointing to the opposite end. Afterward, the magnitude of momentum begins to decrease. When $\tau=\pi$, the average momentum is again zero as shown by "5" which overlaps "1". Then the process proceeds in reverse direction, and repeatedly. When $\tau$ is larger, the periodicity will be gradually spoiled by the interaction. This is shown in Fig. [5b, where "5" overlaps "1" no more. Instead, the peak of "5" shifts a little to the left. It implies that the peak has not yet completely arrived at the opposite end, its arrival is delayed.

The momentum-spin correlation can be revealed by $Q_{\nu}\left(\mathbf{p}, \tau_{c}\right)$. However, we are more interested in a quantity which is easier to be observed. Therefore, we define $Q_{\nu}^{I}\left(\theta_{p}, \tau_{c}\right) \equiv \int p^{2} d p Q_{\nu}\left(\mathbf{p}, \tau_{c}\right)$, where $\theta_{p}$ is the angle between $\mathbf{p}$ and the $+Z$-axis (the azimuthal angle of $\mathbf{p}$ is irrelevant). This is the probability of finding a particle in $\nu$ and emitting at a specified direction $\theta_{p}$ at $\tau_{c}$. It fulfills $2 \pi \int \sin \theta_{p} d \theta_{p} Q_{\nu}^{I}\left(\theta_{p}, \tau_{c}\right)=1$. This quantity is much easier to be experimentally measured because only the orientation of spin is concerned, which can be measured via a Stern-Gerlach device setting towards the outgoing angle $\theta_{p}$.

$Q_{\nu}^{I}$ is sensitive to $\tau_{c}$ (therefore both the time of creation and the time of cancellation of the broad potential should be precise). For examples, when $\tau_{c}=69.5 \pi$ (meanwhile the average positions of both particles are close to the center), $Q_{3}^{I}$ against $\theta_{p}$ with $g_{0}$ given at three presumed values are plotted in Fig. 6a , where the momentum is distributed around $\theta_{p}=0$. Thus the particle is rushing back to the initial end disregarding how $g_{0}$ is (meanwhile the $\nu=-3$ particle is rushing to the opposite end). If $\tau_{c}=70.5 \pi$, the peak of 6 a would appear at the right (not yet plotted). When $\tau_{c}=70 \pi$ (meanwhile the particle is close to the initial end), $Q_{3}^{I}$ depends slightly on $\theta_{p}$ as shown in $6 \mathrm{~b}$, the dependence is not very sensitive to $g_{0}$.

On the other hand, due to the spin-flips, the $|\nu| \neq 3$ components are created and the creation is sensitive to the interaction. For the case of the $\nu=2$ component plotted in 6r, the emission with a smaller $\theta_{p}$ would be more probable if $g_{0}$ is negative, but less probable if $g_{0}$ is positive. The probability $Q_{2}^{I}(0,69.5 \pi)$ would decrease by 7 times if $g_{0}$ is changed from $-g_{6} / 4$ to $g_{6} / 4$. Thus, information on the interaction can be extracted.

When $\tau_{c}$ increases, $Q_{2}^{I}$ would also increase gradually. This is shown in [6k, [6:, and 6f, where $\tau_{c}=2 k \pi-\pi / 2$ (note that the ordinates of these figures have different scales). An example with $\tau_{c}=2 k \pi$ is shown in $6 \mathrm{~d}$. Comparing $6 \mathrm{~d}$ with $6 \mathrm{c}$, one can see how $Q_{2}^{I}$ is changed after the interval $\pi / 2$.

The features of other $|\nu| \neq 3$ components are more or less similar to those of $\nu=2$. Information on the interaction can also be extracted from them. All the curves in Fig. 6 will undergo a left-right reflection if $\tau_{c}$ is changed from $2 k \pi-\pi / 2$ to $2 k \pi+\pi / 2$, or if $\nu$ is changed to $-\nu$. The reflection is nearly exact.

It is emphasized that the dependence on interaction is hardly found in the early stage. For an example, when $\tau_{c}=2 \pi$ (meanwhile both particles nearly completely 


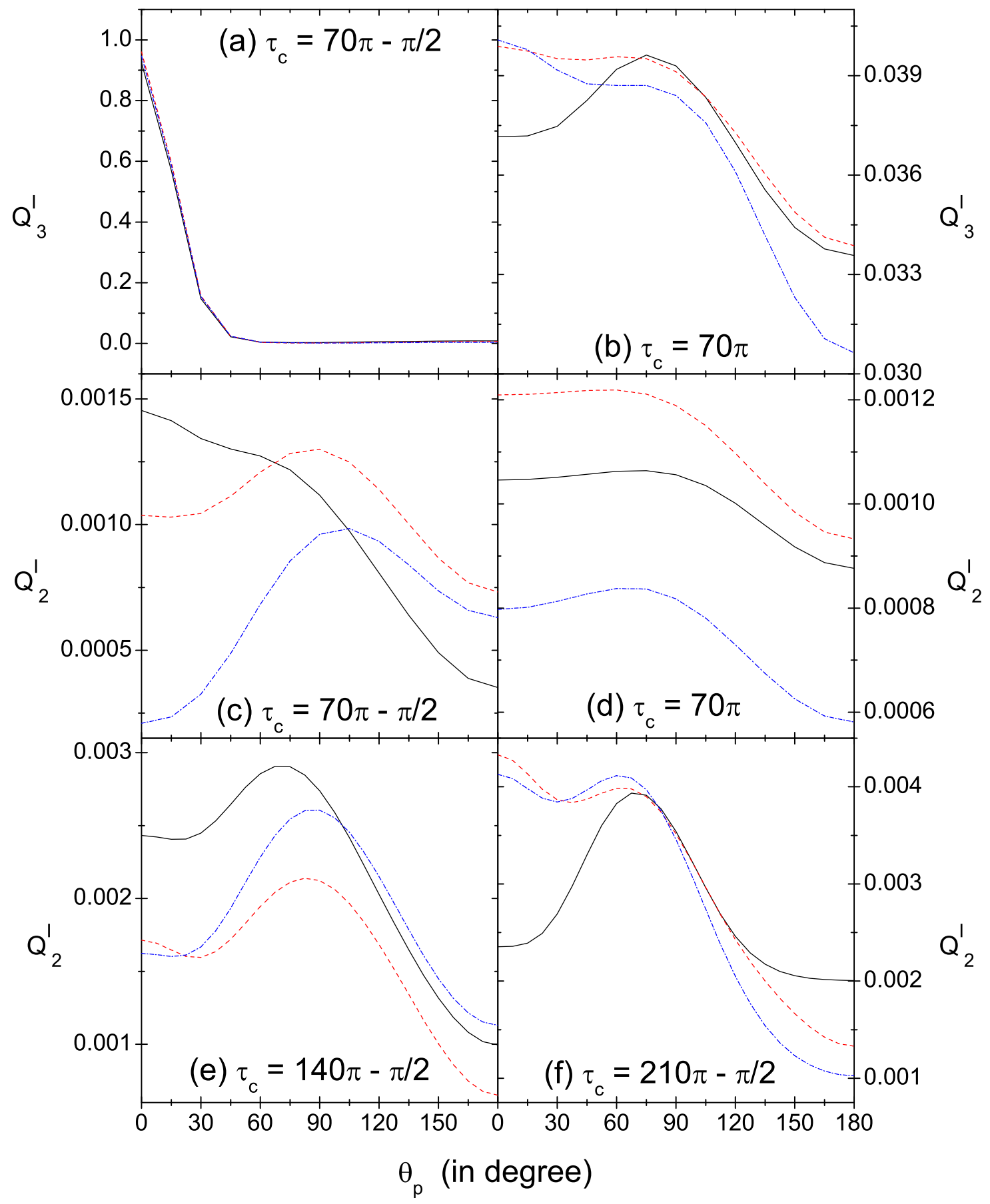

Figure 6. (Color online) Angular distribution of $Q_{\nu}^{I}\left(\theta_{p}, \tau_{c}\right)$ against $\theta_{p} . \nu=3$ for (a) and (b), and $\nu=2$ for the others. $\tau_{c}$ is fixed and marked on the panels. $g_{0}$ is given at three values $-g_{6} / 4,0$ and $g_{6} / 4$, respectively, and the associated curves are in solid, dash, and dash-dot lines. The other parameters are the same as in Fig. 1. 
return to their initial positions after two rounds of collisions), the curves of $6 \mathrm{~b}$ will become a set of horizontal lines and very close to each other, and therefore no valuable information can be extracted. Thus the introduction of the trap, that leads to repeated collisions, is necessary so that the weak effect of interaction can be accumulated.

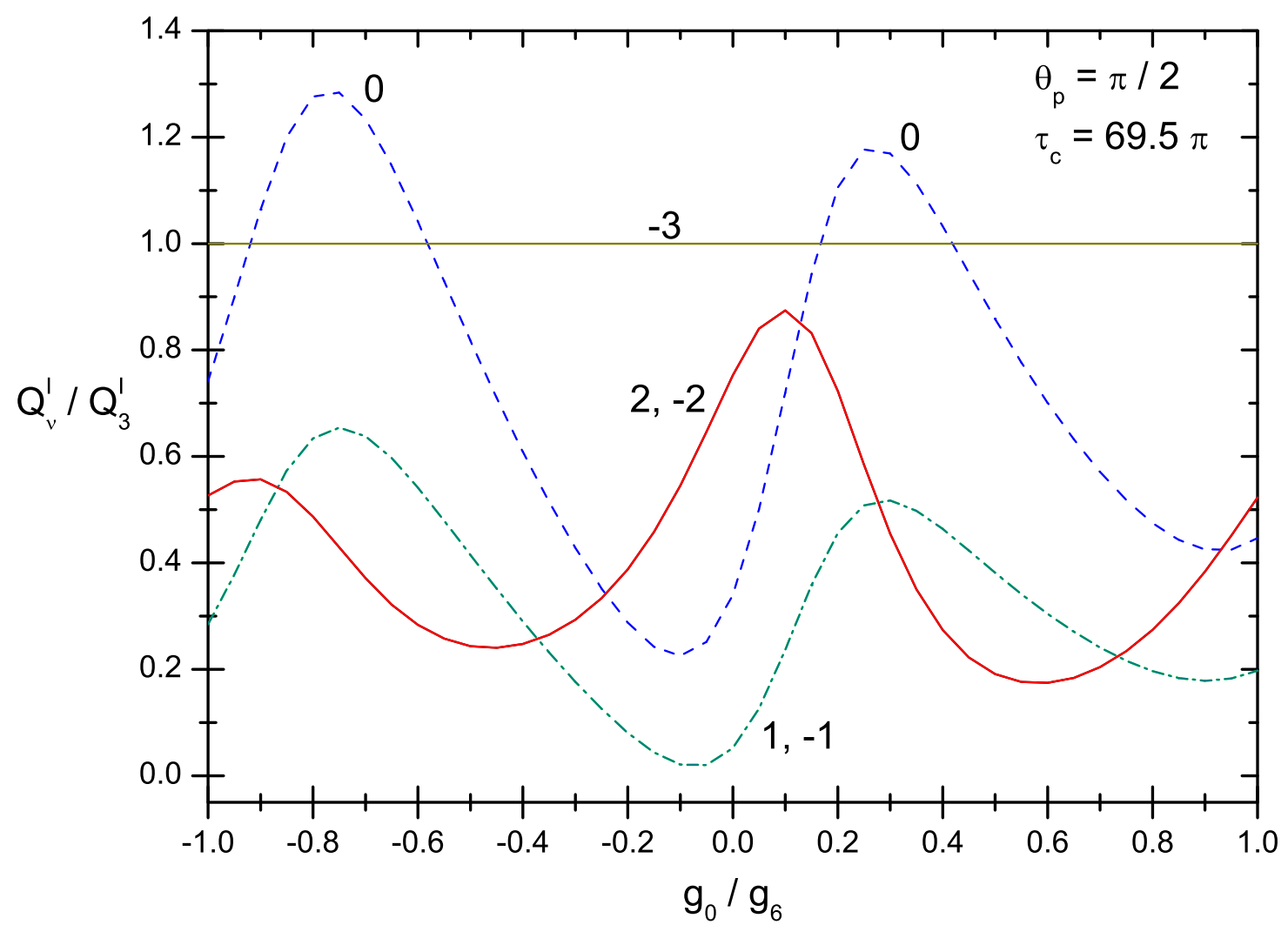

Figure 7. (Color online) $Q_{\nu}^{I} / Q_{3}^{I}$ with $\theta_{p}=\pi / 2$ and $\tau_{c}=69.5 \pi$ against $g_{0} / g_{6} . \nu$ is marked by the curves. The other parameters are the same as in Fig. 1.

When $\theta_{p}$ and $\tau_{c}$ are both fixed, an example is shown in Fig. 7 where the ratio $Q_{\nu}^{I} / Q_{3}^{I}$ with $\theta_{p}=\pi / 2$ and $\tau_{c}=69.5 \pi$ is plotted against $g_{0} / g_{6}$. The curves with $\nu$ and $-\nu$ overlap with each other entirely (however, if $\theta_{p} \neq \pi / 2$, they do not). It is clear from the figure that $g_{0}$ can be uniquely determined if a few ratios can be measured (say, those with $\nu=2$ and 0$)$.

\section{Conclusions}

In summary, an idea is proposed to study the trapped 2-body scattering. The two particles are first localized, then they collide with each other repeatedly in a trap, then they escape. Related theoretical derivation and numerical calculation have been performed to study the coordinate-spin and momentum-spin correlations. The ${ }^{52} \mathrm{Cr}$ atoms have been chosen as an example. Due to the repeating collisions in the trap, the effect of the weak interaction can be accumulated and enlarged, and the strength $g_{0}$ can be thereby determined. It is expected that the approach might open a new way 
for studying various spin-dependent interactions among atoms (molecules) with nonzero spin, in particular for the studies of very weak forces.

\section{Acknowledgments}

The support from the NSFC under the grant 10874249 and from the project of National Basic Research Program of China (2007CB935500) is appreciated.

\section{Appendix A. Matrix elements of $V_{12}$ between the basis functions}

$$
\begin{aligned}
& \left\langle\phi_{n^{\prime} l^{\prime}}(r)\left(l^{\prime} S^{\prime}\right)_{J^{\prime}}\left|V_{12}\right| \phi_{n l}(r)(l S)_{J}\right\rangle \\
= & \delta_{J^{\prime}, J}\left[\frac{1}{4 \pi} g_{S} \delta_{l^{\prime} 0} \delta_{l 0} \delta_{S^{\prime} S} \phi_{n^{\prime} 0}(0) \phi_{n 0}(0)-252 \sqrt{5} C_{d} \sqrt{\left(2 S^{\prime}+1\right)(2 S+1)(2 l+1)}\right. \\
& \left.\left.\cdot C_{1,0,1,0}^{2,0} C_{l, 0,2,0}^{l^{\prime}, 0} W\left(l 2 j S^{\prime} ; l^{\prime} S\right)\left\{\begin{array}{ccc}
1 & 1 & 2 \\
3 & 3 & S \\
3 & 3 & S^{\prime}
\end{array}\right\} \int \frac{d r}{r} \phi_{n^{\prime} l^{\prime}}(r) \phi_{n l}(r)\right], \quad \text { (A. } 1\right)
\end{aligned}
$$

where the Clebsch-Gordan, Wigner and 9- $j$ symbols [15] are introduced. Furthermore, due to the Boson statistics, both $s+l$ and $s^{\prime}+l^{\prime}$ should be even.

\section{References}

[1] D. M. Stamper-Kurn, M. R. Andrews, A. P. Chikkatur, S. Inouye, H.-J. Miesner, J. Stenger, and W. Ketterle, Phys. Rev. Lett. 80, 2027 (1998).

[2] J. Stenger, S. Inouye, D. M. Stamper-Kurn, H.-J. Miesner, A. P. Chikkatur, and W. Ketterle, Nature (London) 396, 345 (1998).

[3] M. Albiez, R. Gati, J. Fölling, S. Hunsmann, M. Cristiani, and M. K. Oberthaler, Phys. Rev. Lett. 95, $010402(2005)$.

[4] A. Griesmaier, J. Werner, S. Hensler, J. Stuhler, and T. Pfau, Phys. Rev. Lett. 94, 160401 (2005).

[5] D. H. J. O'Dell, S. Giovanazzi, and G. Kurizki, Phys. Rev. Lett. 90, 110402 (2003).

[6] L. Santos, G. V. Shlyapnikov, and M. Lewenstein, Phys. Rev. Lett. 90, 250403 (2003).

[7] J. Werner, A. Griesmaier, S. Hensler, J. Stuhler, T. Pfau, A. Simoni, and E. Tiesinga, Phys. Rev. Lett. 94, 183201 (2005).

[8] J. Stuhler, A. Griesmaier, T. Koch, M. Fattori, T. Pfau, S. Giovanazzi, P. Pedri, and L. Santos, Phys. Rev. Lett. 95, 150406 (2005).

[9] A. Griesmaier, J. Stuhler, T. Koch, M. Fattori, T. Pfau, and S. Giovanazzi, Phys. Rev. Lett. 97, $250402(2006)$.

[10] R. B. Diener and T. L. Ho, Phys. Rev. Lett. 96, 190405 (2006).

[11] H. Mäkelä and K.-A. Suominen, Phys. Rev. A 75, 033610 (2007).

[12] S. Uchino, T. Otsuka, and M. Ueda, Phys. Rev. A 78, 023609 (2008).

[13] P. Van Isacker and S. Heinze, J. Phys. A 40, 14811 (2007).

[14] L. Santos and T. Pfau, Phys. Rev. Lett. 96, 190404 (2006).

[15] A. R. Edmonds, Angular momentum in quantum mechanics (Princeton, Princeton University Press, 1957).

[16] S. Y. T. van de Meerakker, P. H. M. Smeets, N. Vanhaecke1, R. T. Jongma, and G. Meijer, Phys. Rev. Lett. 94, 023004 (2005).

[17] J. R. Bochinski, E. R. Hudson, H. J. Lewandowski, and J. Ye, Phys. Rev. A 70, 043410 (2004). 
[18] J. M. Sage, S. Sainis, T. Bergeman, and D. DeMille, Phys. Rev. Lett. 94, 203001 (2005).

[19] D. Wang, J. Qi, M.F. Stone, O. Nikolayeva, B. Hattaway, S.D. Gensemer, H. Wang, W.T. Zemke, P.L. Gould, E.E. Eyler and W.C. Stwalley, Eur. Phys. J. D 31, 165 (2004).

[20] D. Egorov, W.C. Campbell, B. Friedrich, S.E. Maxwell, E. Tsikata, L.D. van Buuren and J.M. Doyle, Eur. Phys. J. D 31, 307 (2004).

[21] W. Tobocman, Nucl. Phys. A. 357, 293 (1981).

[22] M. Baranger, and K. T. R. Davies, Nucl. Phys. 79, 403 (1966)

[23] T. A. Brody and M. Moshinski, Monografias del Instituto de Fisica, Universidad Nacional Autonoma de Mexico (1960). 